\title{
Molecular biologists in Asia and Pacific plan research network
}

[TOKYO] Life scientists from more than 20 institutions in the Asia-Pacific region met in Tokyo last Saturday (28 June) to discuss creating a network of researchers and institutions to promote collaboration in molecular biology and biomedical research.

Supporters of what has tentatively been called the International Molecular Biology Network (IMBN) for Asia and the Pacific Rim hope that it may eventually play a similar role to that of the European Molecular Biology Organization (EMBO).

The network is the brainchild of researchers at the Institute for Molecular Biology and Genetics at Seoul National University in South Korea, and the Institute of Medical Science of Tokyo University, where the meeting was held. Participants also included scientists from China, Taiwan, Hong Kong, Singapore, Malaysia, Australia, and New Zealand.

Sunyoung Kim of the Seoul institute, one of the organizers of the meeting, says that he hopes the network will awaken interest in molecular biology in parts of Asia where the subject is still in the early stages of development.

Cooperation between institutions in Asia is at present limited for complex historical, political and geographical reasons, and links

tend to be stronger with the United States, where much of the innovation in the field occurs. But collaboration between Kim's institute and Tokyo University over the past four years has convinced researchers at both institutions of the value of intraregional cooperation.

Other participants at the meeting were equally enthusiastic about improving links through regional postdoctoral fellowship schemes, conferences, workshops, shortterm visits and practical courses. There was even talk of establishing a regional laboratory or laboratories, similar to the European Molecular Biology Laboratory in Heidelberg, Germany, which was set up by EMBO.

But there was no consensus on the form the network should take, or on its initial goals. For example, a draft document circulated at the meeting proposed two levels of membership, institutional and individual. But participants from China expressed reservations about a system based on institutions, without broad national and government backing.

The Chinese scientists proposed instead that branches be set up in each country to draw support from all institutions and the government. "If only one or two institutes from China join, others will quickly lose

\section{Promising signs for NSF budget increase}

[WASHINGTON] A key subcommittee of the US House of Representatives has proposed a funding increase for the National Science Foundation (NSF) of almost 7 per cent, suggesting that the agency could receive a substantial cash boost next year.

The foundation would get an extra $\$ 90$ million to rebuild its research station at the South Pole, in the proposal agreed by the appropriations subcommittee chaired by Jerry Lewis (Republican, California).

The subcommittee's proposal has still to be passed by the full Appropriations Committee and the entire House, and then reconciled with a yet-to-be-determined Senate proposal. But the decision has already raised the hopes of scientific societies in Washington, which had called for a 7 per cent increase for the NSF earlier this year (see Nature 386, 7; 1997).

Officials of these organizations say the decision reflects a growing acceptance in the Congress that politicians will get credit for investing money in science - and indicates how the scientific community can have an impact on the process. "When people raise

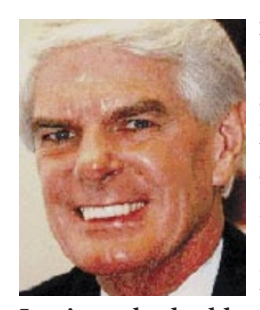

issues, the people in Congress do pay attention," says Mike Lubell, head of public affairs at the American Physical Society.

Under a bill passed by Lewis's subcommittee on Lewis: seeks double 17 June, the NSF would Clinton increase. \section{get $\$ 3.5$ billion in the} 1998 financial year which

starts on 1 October next -6.6 per cent more than last year, and just over twice the increase requested by the Clinton administration in February (see Nature 385, 569; 1997).

Most of the extra money would go to the Antarctic programme. But money for research in universities would also be increased by 4.3 per cent. The equivalent Senate subcommittee, which has been given a smaller allocation of money than Lewis's, is expected to offer less to the NSF. But advocates of the agency say they hope it will propose an increase greater than the anticipated rate of inflation. Colin Macilwain interest," said Wei-Feng Chen of the department of immunology at Beijing Medical University.

There were also divergent views on individual membership. The draft proposed that any interested individual could join. But Frank Gannon, the executive director of EMBO who acted as an adviser to the meeting, pointed out that some degree of selection was essential to achieve scientific 'excellence'.

By the end of the meeting the participants were leaning in favour of a system of selective individual membership, where each participant, perhaps aided by neutral advice from EMBO, will nominate between five and ten outstanding scientists from their country, but allowances will be made in developing countries for the definition of 'outstanding'.

Also unresolved is the source of funding. The meeting was attended by senior officials from Japan's Ministry of Education, Science, Sports and Culture, the Science and Technology Agency and the Ministry of Foreign Affairs, as well as officials of the Korea Research Foundation, which is affiliated to South Korea's education ministry. They voiced support for the concept of IMBN but did not discuss where funds might come from, and could only point to existing or soon-to-be launched programmes that indirectly support the goals of the network.

Some participants expressed fears that, if only one or two countries provide funds for the network, they might dominate its activities. But Ken-ichi Arai, chairman of the department of molecular and developmental biology at Tokyo University's Institute of Medical Science, and an organizer of the meeting, believes that once the network is more clearly defined it will be possible to approach the Asia-Pacific Economic Cooperation (APEC) forum for support.

APEC, whose members include all the Asia-Pacific countries represented at the IMBN meeting, recently initiated meetings of its science ministers to develop intraregional cooperation in science and technology (see Nature 384, 200; 1996), and the new network is closely aligned with APEC's goals.

One proposal discussed at the meeting was the setting-up of a website to link institutions, as an easy first step to develop the network. The meeting closed with a decision to set up a task force with seven members from Japan, Korea, Taiwan, China, Hong Kong, Singapore and New Zealand to seek 'seed' money of about \$2 million from various sources in order to run the network for the first one to two years.

DavidSwinbanks 